\title{
Design Philosophy and Methods of Alvar Aalto from the Ecological Aesthetics
}

\author{
Jun Du \\ School of Architecture and Design, Sothwest Jiaotong University, Chengdu, China
}

Email address:

dajundu@aliyun.com

To cite this article:

Jun Du. Design Philosophy and Methods of Alvar Aalto from the Ecological Aesthetics. International Journal of Literature and Arts. Vol. 7, No. 4, 2019, pp. 87-92. doi: 10.11648/j.ijla.20190704.13

Received: July 17, 2019; Accepted: August 26, 2019; Published: August 27, 2019

\begin{abstract}
This work aims to study the design ideas of Alva Aalto from the perspective of ecological aesthetics. He is a famous Finnish architect and industrial designer, as well as an internationally renowned modernist design master. He absorbs the modernist design concept objectively and critically, and he adheres to green design, organic design and pays attention to the physical and psychological experience of users. Aalto's design clearly reflects the idea of ecological aesthetics and breaks through the time limit, making his design ideas still have important value to guide today's design.
\end{abstract}

Keywords: Alvar Aalto, Ecological Aesthetics, Design Philosophy

\section{Introduction}

At the beginning of the 20th century, the wave of industrialization brought about the scientific and technological achievements, but also caused a lot of problems: the outbreak of ecological crisis, the indifference of product design and the lack of humanistic care. Faced with these increasingly serious problems, people are increasingly longing for the return of humanity and nature, and the concept of ecology has been more and more widely understood and recognized. As early as 1866, the German biologist Haeckel put forward the concept of "ecology". In the late 1980s, with the rapid development of ecological discipline and its gradual penetration into other related disciplines, ecological aesthetics began to take shape gradually. Ecological aesthetics is an organic combination of ecology and aesthetics. It studies aesthetics with the theories and methods of ecological aesthetics, and also examines the ecological environment with the vision of aesthetics, thus forming a brand new aesthetic theoretical form [1]. Only by understanding the interaction between nature and society can we create design products that meet the aesthetic needs of both users and designers, and at the same time make artistic design products have enduring life.

Alvar Aalto respects nature and advocates humanized design, of which the starting point is the user's physical and psychological experience. Between the 1920s and 1970s, he designed many architectural works and a large number of furniture, glass and lighting designs that embodied the idea of ecological aesthetics [2]. In recent years, domestic and foreign scholars have explored the ecological factors in Alva Aalto's design. Chen Wang, Jun Du et al. [3] believe that in the course of his designing Aalto not only considers users' comfort and safety requirements from the perspective of product functions, but also considers the close connection between users and the nature, thus empowering products with ecological aesthetics. Jun Du, Fuye Zhang et al. [2] point out that Aalto's design is no longer an aesthetic object independent of users, but an equal existence of interaction and symbiosis between users and products. Hyon-sob Kim [4] believes that Aalto's architectural design coordinates the relationship between human and nature and returns to ecology, with eternal value. Aalto designed in a natural manner, and in his designs he strives to blur the boundaries of human life and the natural environment [5]. He pioneers a design direction of ecological aesthetics. As the Italian designer Gianluca Gelmini analyzed, "Alvar Aalto does not simply seek for the simple balance and harmony between architecture and nature... It's about incorporating nature inside and outside of the building... ", "he also imitates the structure of natural order and control biological mechanism to explain the form of architecture, 
space, and the relationship between architecture and city", and Gelmini further pointed out that Aalto makes the relationship between nature and biology the most prominent part of the topic of the relationship between human and environment [6]. To analyze and research Aalto's design from the perspective of ecological aesthetics is conducive to the summary of his design thoughts, and expand the design thinking mode in the field of aesthetics, so as to promote the exploration of modern Chinese design concepts.

\section{Design Direction Based on Ecological Aesthetics}

Alvar Aalto inherits the Nordic people's view of nature and the cultural view of "beauty for daily life", while integrating the technical view of modernist design, as Figure 1. His design emphasizes organic form, and adopts natural materials such as wood and red brick [7], and insists on exploring the best combination of design and nature, highlighting strong ecological aesthetic consciousness and humanistic care.

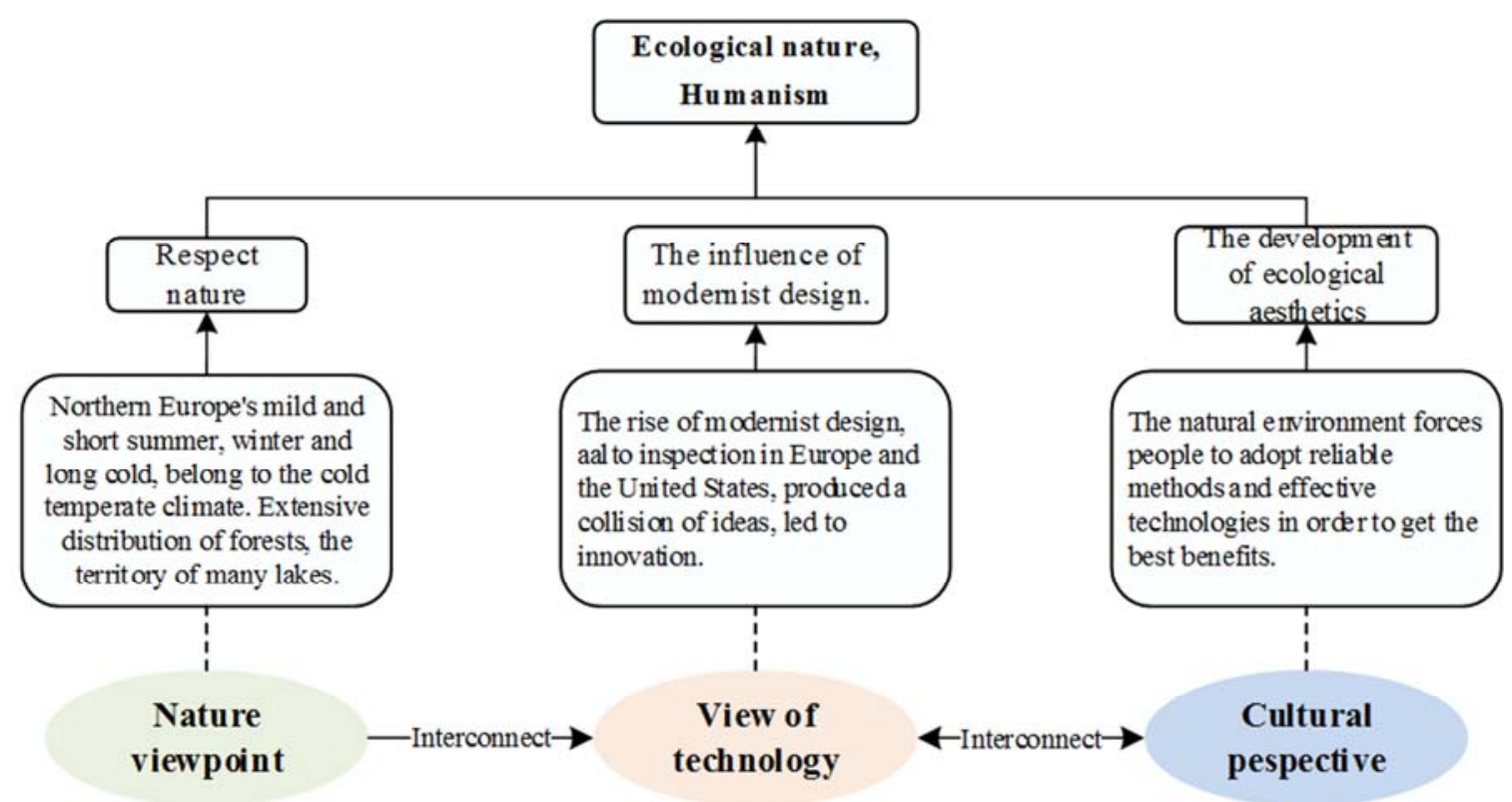

Figure 1. Impact of Nordic views on nature, humanity and technology on Alvar Aalto.

\subsection{Inspiration from the Natural Environment}

The ecological aesthetic thought contained in Aalto's design embodies the respect for humanity and naturalism. The formation of this design idea is not accidental. First, Aalto was born in Finland in Scandinavia, where there are many lakes and natural elegance. But in the far north of Europe, the sun is very short. Because of Finland's unique geography, he shares most scandinavians' love of nature. Secondly, in the cold climate of Scandinavia, people have long created tools adapted to nature through traditional handicraft design, which has penetrated into their daily life and social interaction. For a long time, the Finnish people have lived a self-sufficient life. The exquisite craftsmanship and the design concept of "emphasizing utility" have been kept intact.

\subsection{Influence of Modernist Design Concepts}

Since the 1920s, Aalto has been traveling in Europe and America, witnessing the development and rise of modernist architecture. He participated in numerous design projects at home and abroad. Aalto was also guided by Swedish designer Gunnar Asplund, who admired nature. At the same time, he got on well with Eliel Saarinen, the pioneer in the field of design, Eero Saarinen, Corbusier and Frank Wright, and forged a deep friendship with Picasso, the great artist, and
Laszlo Moholy nagy, the master of Bauhaus constructivism. On the way of exploring design, Aalto always puts himself at the forefront of design through design practice and communication with pioneers. All these experiences and feelings contribute to the maturity of Aalto's design ideas. In the process of thought exchange and design practice, Aalto realized that although modernism endows product design with more technology and new materials, it makes people and nature more and more distant in the way of life.

\subsection{Development of Ecological Aesthetics}

Aalto advocates the design of organic form and human touch, but he is definitely not anti-technicalism, but serves the concept of ecological aesthetics and technology in design. There are various kinds of beauty in nature, and the designer must have the ability of aesthetic appreciation to realize its value [8]. Only by seeking, discovering and collecting various peculiar life forms in nature can designers induce creation opportunities and establish the theme of works [4]. Aalto forms a state of returning to nature in design. He pays attention to the integration of design ideas and natural environment, forming three major features in design: wavy curve, irregular fan-shaped or radiating plane and stepped plane [9]. In materials, he favors natural materials. Aalto's pursuit of the form of natural beauty plays an important 
leading role in his design, which also reflected the Scandinavians' people's yearning for nature, the combination of nature and products, and the important idea that human beings should be close to the nature. This idea makes Aalto's design work have the basis of ecological aesthetics.

\section{Ecological Aesthetics in Design Practice}

Man is an organic part of the natural ecosystem, and there is an internal harmony between the elements of nature and Man [10]. Aalto presents the gravity and purity of nature in terms of color, material and sense of space, and advocates that design should start from users rather than designers themselves. His designs combine traditional Finnish culture with industrial mass production, combining modernist reason with Scandinavian romance. At the same time, Aalto not only emphasizes the high rationality and practicability of products, but also injects rich natural factors and humanistic emotions into the design. Therefore, his design forms a special modernist design style that is different from Bauhaus's "geometric spirit", machine aesthetics' indifference and rigidity. Critics call it "organic functionalism." In order to facilitate the research, this paper unifies Aalto's achievements in many design fields and analyzes them with the concept of "product".

\subsection{Integration of Ecological Aesthetics and Design}

The ecological beauty expressed by Aalto's works is not the natural beauty in the general sense, but the new design form is generated by the integration of nature and science and technology. The so-called ecological beauty refers to an aesthetic form between natural beauty and society, which comes into being after the natural beauty and social beauty have been fully developed. But formally speaking, ecological beauty is more focused on natural beauty. Its form is mainly about "truth" and the purpose of human "goodness" conforms to the laws of nature itself [11]. Ecological beauty is the product of the harmonious relationship between man and nature. To realize the transformation of products from technological beauty to natural beauty, people must be considered, while ecological aesthetics is the guiding ideology. We can find the inherent nature of Aalto's design works through the appearance of his design works. His architectural design works are simple and natural, with full consideration of ecological and environmental factors, and emphasize the emotional support of users, as well as the communication between human and nature. The natural attribute of ecological products contains the ecological concept of the co-participation of human and nature, and the harmonious coexistence of human and nature. Ecological aesthetics plays a very important role in Aalto's design.

\subsection{Specific Application of Ecological Aesthetics}

Aesthetics of ecological beauty requires artistic works to express both formal beauty and content beauty, and the formal beauty and content beauty achieve organic unity in the aesthetic process [12]. The ecological aesthetic thoughts contained in Aalto's works are expressed through the image, space combination, decoration, texture and color of the design works, as well as the principles of ecology, functionality, practicality, comfort and economy of the design objects. Aalto interprets the idea of ecological aesthetics with his own design techniques, unifying "formal beauty" and "content beauty" in the design works.

\subsubsection{The Architectural Design}

Studying the architectural works of Alvar Aalto, we will find that they have already embodied the idea of ecology. Aalto believes that architecture and the biology of nature are inextricably linked. In order to achieve harmony between man and nature, he designs the surrounding ecological conditions, ventilation, lighting and other elements reasonably and respected the original ecological structure to create an appropriate ecological system for the buildings, so that the space of the buildings will form a breathing and growing ecological system.

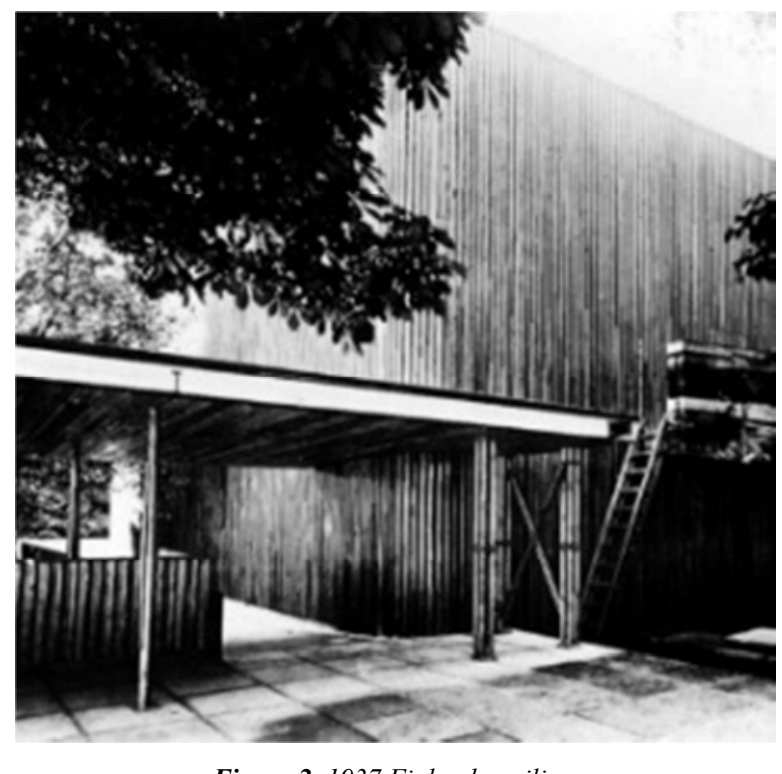

Figure 2. 1937 Finland pavilion.

(1) Aalto has a special love for wood. He gives full play to the natural properties of wood, and skillfully integrates the warmth and life characteristics of wood into his design works. At the same time, he combines organic modeling with wood to create simple and elegant buildings that return to nature. Aalto's Finnish Pavilion for the international exposition in Paris in 1937 was his first work to attract much international reaction. The pillars of the Finland pavilion are made of logs bound with rattan, and the external walls are spliced with wooden boards. With the courtyard as the core, the exhibition halls are connected together, and each space gets good natural lighting. The building is placed in a dense shade, and the overall size is small, presenting a free and elegant shape, as Figure 2. Aalto uses organic modeling to design the exterior wall of the Finland pavilion and the wall of the internal courtyard into a zigzag modeling, which expresses the rebellion against the indifferent geometric rules. 
(2) Aalto can flexibly deal with architectural functions and layout according to the needs of the natural environment, so that people can interact with the living environment more freely and have a freer lifestyle. On January 1, 1939, Aalto completed all the design drawings of Villa Mairea, which was his most outstanding work and marked that Aalto's design thought had completely stepped out of the confinement of functional aesthetic standards. The plan of the house is L-shaped, with a combination of clear brick walls, plaster walls and wooden walls; He designed a flowing space on the ground floor, integrating the gallery, living room, dining room, etc. The division of the space areas depends on the decorative features, as Figure 3. Due to the use of natural materials, this large open space feels warm [13]. The fluidity of the space connects the indoor and outdoor environment closely.

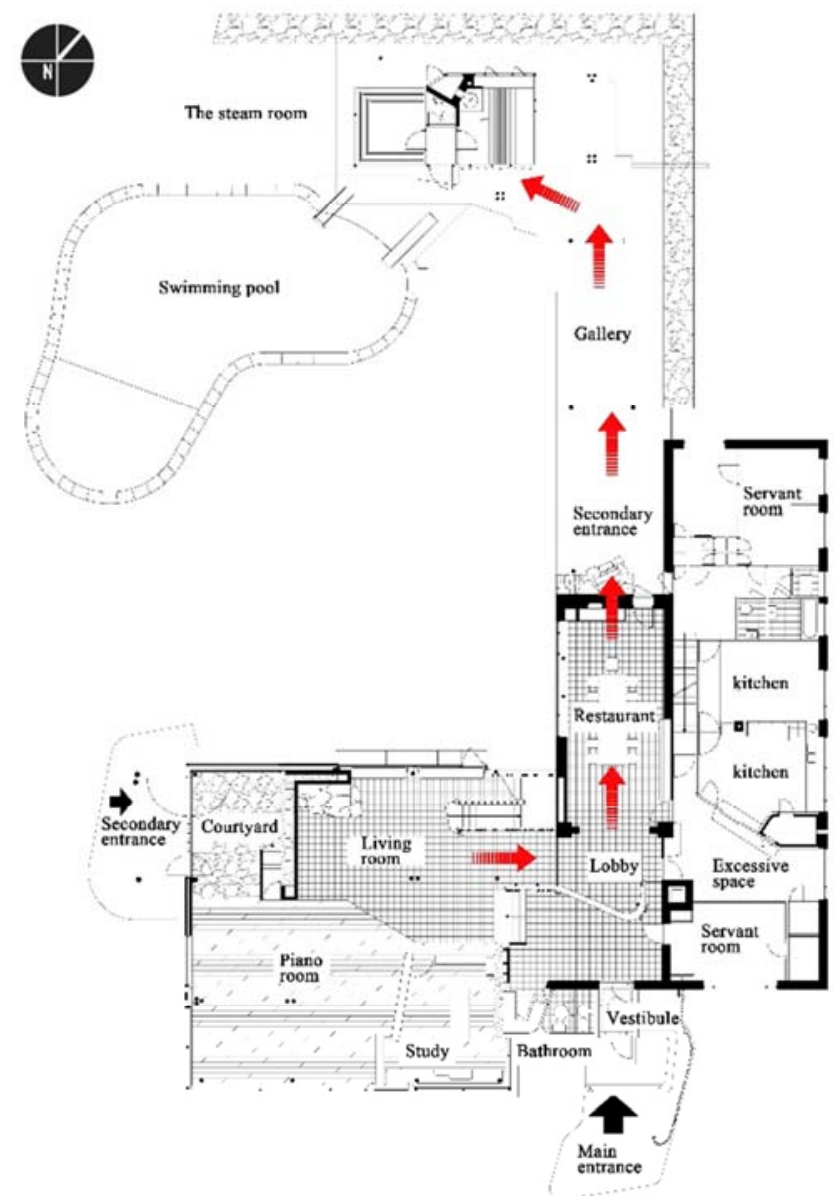

Figure 3. Plan of villa Mairea.

\subsubsection{Furniture Design}

Aalto has made innovation and exploration in laminating and gluing technology to produce curved wood furniture. In the 1920s, Aalto and his wife, Aino Marsio, took native Finnish birch and bent the wood using organic forms. The experiment, which lasted five years, eventually allowed Aalto to design a series of landmark chairs. Inspired by Marcel Breuer of Bauhaus who made Wassily chairs out of steel tubes, Aalto designed the "Paimio" armchair in 1931. From the perspective of ergonomics, Aalto hot-pressed plywood into seats and backrests, which broke through the indifferent geometric form of modernism in structure and adopted the dynamic curve, as Figure 4. Mr. and Mrs. Aalto modified the cantilever chair designed by the modernist design master Mies with steel pipes. In 1932, they designed the easy chair No. 42, which was the world's first plywood cantilever chair [14], as Figure 5. Its seat hangs over the top of the "C" shape. Aalto's insistence on wood is an expression of the Nordic design community's meditation and revision of the cold modernist design.

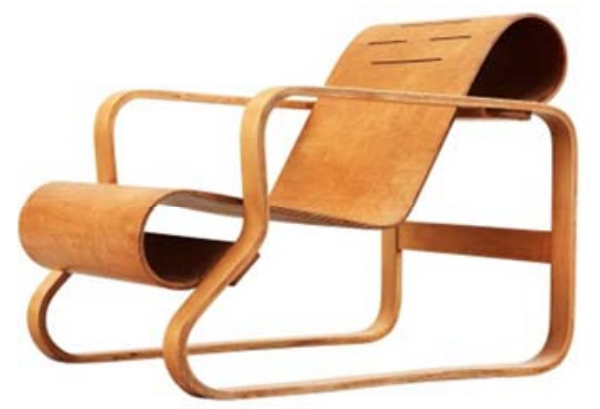

Figure 4. Pamio armchair.

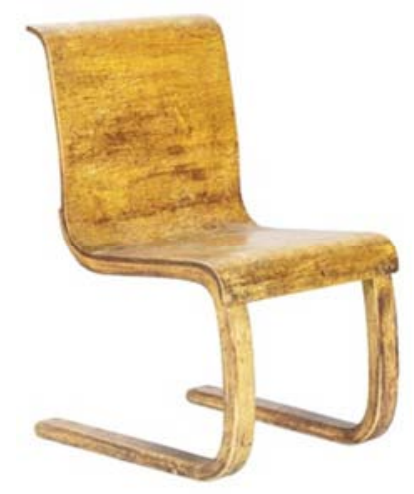

Figure 5. Cantilever chair 42.

\subsubsection{Glass Design}

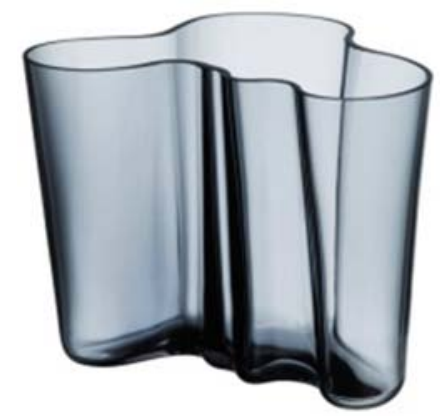

Figure 6. Savoy.

Alva Aalto not only advocates to learn from nature in glass design, but also pays attention to material selection. He extracts national characteristics from traditional Finnish culture as the core elements of the design, perfectly combining organic forms and glass materials, and endows the 
design works with natural features and rich connotations. The products designed by him not only focus on the users' satisfaction in function and physical comfort, but also emphasize the spiritual pleasure, allowing users to perceive the natural form through visual perception and tactile perception of material texture. In 1936, Aalto and his wife Aino designed the Savoy series of vases, as Figure 6. It breaks through the concept of bottle rule in form, and its design is inspired by the winding lake boundary line in Finland. It adopts wavy curve to express the elegant and graceful character, and its shape is lively and fresh. Aalto pursues the modern product design of natural ecological aesthetics. The bionic products in the shape emit the natural taste and the bionic functions make people close to life. The innovation of glass design comes from Alvar Aalto's understanding and interpretation of the harmony between man and nature.

\subsubsection{Design of Lamps and Lanterns}

Aalto attaches great importance to the design concept of integration and regards lights and chairs as part of the indoor environment. In 1939, Aalto designed the Golden Bell Light chandelier for the famous Savoy Restaurant in Helsinki. The shell is Golden and the shape is similar to the hanging Bell, so it is called "Golden Bell" chandelier. It is hung in the Restaurant with a 3-meter long line, and the shape is extremely simple, as Figure 7. Lighting design is a part of the overall design of savoy restaurant. And "admiralty" pendant lamp received people's keen attention at that time, for which the main reason is that the design of lamps and lanterns at that time was in the exploration period, the design of this lamps and lanterns of Aalto broke through the influence of neoclassical style, elegant and rich in modern sense, bringing a new aesthetic perspective. The lamp shape has a unique curved change, its manufacture craft is exquisite, and uses the diffuse reflection illumination, the light is mild, is different from the indifferent modernism design. Up to now, "golden bell" droplight still has a strong artistic quality. The design of lamps and lanterns of Aalto is simple and without redundant decoration, which is not only suitable for mass production, but also reduces the production cost and conforms to the concept of ecological design.

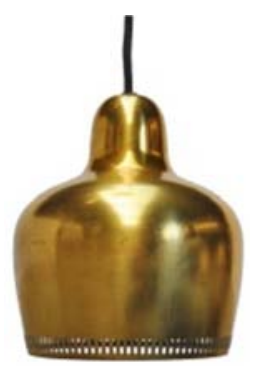

Figure 7. Golden bell.

\section{Design Methods of Aalto's Ecological Aesthetics}

Product design is not only restricted by man-machine relationship and technical factors, but also influenced by the designers' own culture, psychology and emotions. As mentioned above, the design form of Aalto does not exist in isolation, and his modeling is not a purely physical change process, but a process of gestation and growth with vitality. In essence, design modeling activity is a growing process that follows "cultural concept $\rightarrow$ inner inspiration $\rightarrow$ restriction of natural and social laws $\rightarrow$ rational choice" [15], just like a "seed" buried deep in cultural soil, inspired by the external environment and bursting into creative inspiration. Jun Du, Fuye Zhang et al. [2] believes that the maturity of Aalto's design ideas has gone through the process of creative germination, exploration, integration and generation. First, Finland's view of nature, culture and technology give birth to the seeds of Aalto's design inspiration. Second, seeds are stimulated and grow by external environment (social system, technological reform, design trend of thought, etc.); Third, through the exploration of "culture layer, behavior layer and reflection layer", combined with the re-processing of modeling relations (morphological expression, spatial relations and environmental relations), the modeling can be obtained. Fourth, products with an affinity and natural nature that are innovative and quiet are finally formed, as Figure 8. At the same time, Aalto's design idea is a thinking line that follows the cycle of "divergence -- cohesion -- divergence" and reflects the design concept different from other design masters: technology is a means for people to enjoy and use products and get joyful experience.

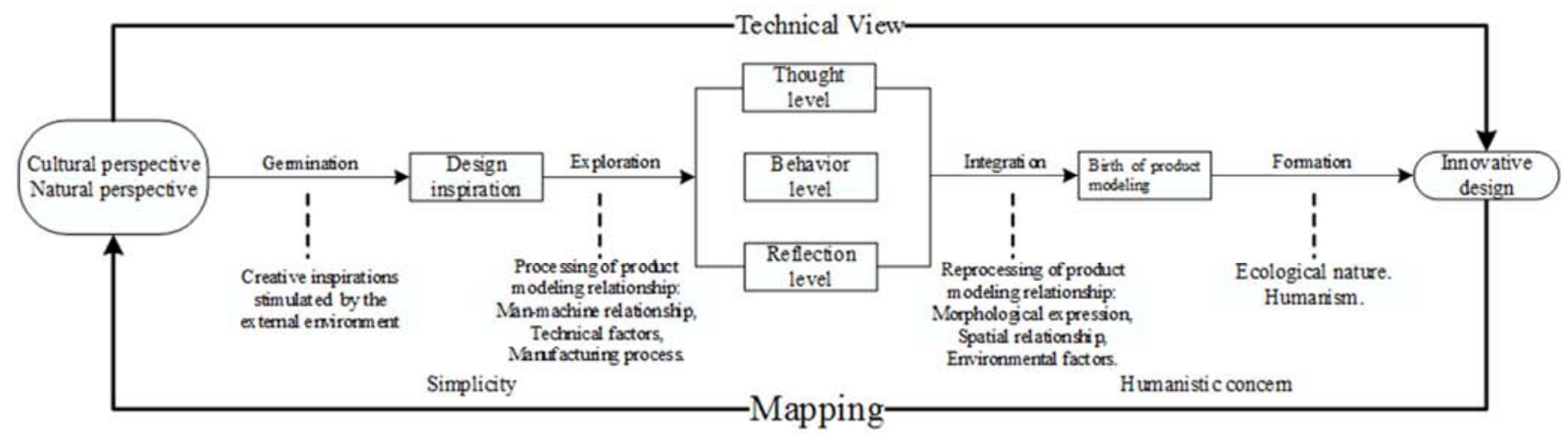

Figure 8. Analysis of Aalto's ecological aesthetic design. 
Based on the synaesthesia of life is the premise of designer's creation, and cannot be separated from the audience's self-consciousness [16]. Aalto's design has influenced the audience's visual aesthetics and perception experience with the emotional design techniques of visual details and scene atmosphere. Through the image transformation of the original life experience, he inspires and satisfies the aesthetic needs of the audience and innovates the modernist design concept. According to the hierarchy of needs of American psychologist Abraham H. Maslow we can find that Aalto applies natural elements in product design. His design transcends the differences of cultures, which not only in material meets the users' demand, but also triggers the user's "synaesthesia" life, having the consumer's emotional resonance. So Alvar Aalto gains enduring fame and interest all over the world.

\section{Conclusion}

The foundation of ecological aesthetics is human's life experience of the integration of nature, which comes from the union of heaven and man, the integration of heaven, earth, nature and heart, and constitutes a beauty of artistic conception and rhythm in space and time [3]. Alva Aalto, the world-renowned pioneer of modernist design, finds beauty from nature and integrates the inspirations and inspiration brought by nature into his design, so that his design works show the characteristics of ecological aesthetics [17]. The ecological aesthetic significance of Aalto design is as follows: on the one hand, it reflects the close relationship between human and nature in the material, modeling and function of products; on the other hand, the design shows the harmonious coexistence between human and nature. Ecological aesthetics emphasizes the balance between man and nature, and expresses the relationship between man and nature depending on and supporting each other. Aalto's design has gone beyond the time limit. Even today, we can still feel the presentation of this design idea when we look at his works. From the perspective of ecological aesthetics to explore Alvar Aalto's design idea, it promotes the present stage of China's design aesthetic and sustainable development problems, and at the same time, it provides a very valuable reference for us to understand the modernist design, and also provides useful information for us to understand the background of the historical evolution of design.

\section{Acknowledgements}

Sichuan modern design and culture research center project (MD17E016).

\section{References}

[1] Dehua Li, Ecological Design, 1st ed, Beijing: Peking university press, 2012, pp. 60-61.

[2] Jun Du, Fuye Zhang, Bochu Xu, Jin-yi Zhi, Xiaofei Xu, Design Philosophy and Methods of Alvar Aalto from the Cultural Ecology Perspective. Packaging Engineering, vol. 40, pp. 17-222. April 2019.

[3] Chen Wang and Jun Du and Shujing Ren, Alvar Aalto's Furniture Design Based on Aesthetic Psychology. Packaging Engineering, vol. 38, pp. 16-20. August 2017.

[4] Hyon-Sob Kim, Alvar Aalto and Humanizing of Architecture, Journal of Asian Architecture and Building Engineering, 8:1, 9-16, DOI: $10.3130 /$ jaabe. 8.9

[5] Fan Song, Exploration on Alvar Aalto's Architectural Curves in Space Performance. Zhuangshi, vol. 300, pp. 084-085. April 2018.

[6] (Italia) Gianluca Gelmini, Alvar Aalto, Baoquan Wang, 1st ed, Dalian: Dalian university of technology, 2008. pp. 21.

[7] Karl Fleig. Alvar Aalto (Works and Projects) Voulom I. Basel: Birkhauser Verlag, 1995, pp. 9.

[8] Dehua Li, Ecological Design, 1st ed, Beijing: Peking university press, 2012, pp. 74.

[9] (Finland) Goran Schildt, Alvar Aalto: Design excellence, Jie He and xinxin Chen, 1st ed, Bejing: China architecture \& building press, 2005 , pp. 9 .

[10] Yanzu Li, Environment art design, 1st ed, Beijing: China renmin university press, 2005, pp. 359.

[11] Junmin Wang and Jin Yang, Ecological Aesthetics and Product Design, Art and Design, Vol. 4, pp. 194-195.

[12] (Finland) Goran Schildt, Alvar Aalto: Design excellence, Jie He and xinxin Chen, 1st ed, Bejing: China architecture \& building press, 2005, pp. 48-61.

[13] Karl Fleig. Alvar Aalto (Works and Projects) Voulom III. Basel; Birkhauser Verlag, 1995, pp. 220.

[14] Duoduo Zhang, Illustration Nordic design, 1st ed, Wuhan: Huazhong university of science \& technology press, 2019, pp 87.

[15] Bochu Xu and Yang Li, Rail Car Styling Design, 1st ed, Beijing: Chinese Science Press, 2012, pp, 116.

[16] Fan Yu. Bionic Design's Philosophy and Trend. vol 240, Beijing, zhuangshi, pp. 25-27, April 2013.

[17] Hengchun Xu, Ecological Aesthetics, 1st ed, Xian: Shanxi people's education press, 2000, pp. 137. 